\title{
Calibration of RF Field Strength Meters
}

\author{
J. P. M. de Vreede, G. M. Teunisse, K. Münter, H. Hoitink, K. Holland, M. O’Dwyer, L. Bergsten, and L. Puranen
}

\begin{abstract}
An intercomparison of field strength meters was performed during 1991-1993 over the frequency range $100 \mathrm{kHz}-1000$ MHz and field strength range 1-10 V/m using different calibration techniques. Four field sensors, electric as well as magnetic, have been used. Seven institutes participated in this intercomparison using at least three different calibration methods. A calibration uncertainty of less than $1 \mathrm{~dB}$ can be obtained if certain procedures are followed. The results are applicable in the fields of EMC testing and health care (nonionizing radiation).
\end{abstract}

\section{INTRODUCTION}

$\mathbf{O}$ VER several decades, the electronic industry has become familiar with the field of EMC. Comparing different immunity testing facilities, large discrepancies in the field strengths measured have been found. These discrepancies might be explained by the use of comparable, but different (i.e., in size) setups of the field-generating facilities, as well as by the different methods in use for calibration of field strength meters. Therefore, if the uncertainty in the calibration of these field probes could be reduced, this would have a beneficial effect on EMC immunity testing.

This paper describes an investigation that was carried out under the auspices of the Community Bureau of References (BCR, part of the European Communities Commission) in connection with the recent implementation of the $\mathrm{EC}$ directive on EMC. An extensive report is given in [1].

The measurement results are evaluated with emphasis on the calibration procedures being used by (accredited) calibration facilities and national standards laboratories, their quoted uncertainties, and the level of consistency. In this way, the results should make it possible to state an accuracy level in the test procedures for certification, and should be used in the writing of new standards.

\section{Organization}

NMi Van Swinden Laboratorium was appointed to act as the organizing laboratory to purchase the field strength meters

Manuscript received July 1, 1994; revised October 15, 1994

J. P. M. de Vreede and G. M. Teunisse are with the NMi Van Swinden Laboratorium, Postbus 654, 2600 AR Delft, The Netherlands.

K. Münter is with the Physikalisch-Technische Bundesanstalt, Postfach 3345, D-38023 Braunschweig, Germany.

H. Hoitink is with EMC Baden Ltd., c/o ABB Research Centre, CH-5405 Baden-Dätwill, Switzerland.

K. Holland is with the National Physical Laboratory, Teddington, Middlesex, England TW11 0LW.

M. O'Dwyer is with Radio Frequency Technologies, 40 Marrowbone Lane, Dublin 8, Ireland.

L. Bergsten is with the Swedish National Testing and Research Institute, Box 857, S-50115 Borås, Sweden.

L. Puranen is with the Finnish Centre for Radiation and Nuclear Safety, P.O. Box 14, FIN-00881 Helsinki, Finland.

IEEE Log Number 9408947. and to carry out an evaluation. The official measurements were carried out from August 1991 until February 1993. The round-robin intercomparison was carried out as a threeloop circulation with two check measurements at the pilot laboratory.

The following organizations participated.

1) EMC Baden in Dättwill, Switzerland: an independent test and research facility without specific calibration facilities;

2) Radio Frequency Technologies (RFT) in Dublin, Ireland: an independent testing facility without specific calibration facilities;

3) The Swedish National Standards and Testing Institute (SP) in Borås, Sweden: the national standards laboratory for most of the SI units;

4) The Finnish Centre for Radiation and Nuclear Safety (STUK) in Helsinki, Finland: the national authority responsible for the regulation of radiation protection and nuclear safety in Finland;

5) The Physikalisch-Technische Bundesanstalt (PTB) in Braunschweig, Germany: the national standards laboratory and center of metrology in Germany;

6) The National Physical Laboratory (NPL) in Teddington, England: the national standards laboratory and center of metrology;

7) NMi Van Swinden Laboratorium (VSL) in Delft, The Netherlands: the national standards laboratory.

At the time of the project, the last three laboratories were regularly issuing calibration certificates for probes similar to those used in the intercomparison.

\section{TRANSFER STANDARDS}

Generally speaking, the immunity testing levels range from 1 up to $10 \mathrm{~V} / \mathrm{m}$ in the frequency range $0.1-1000 \mathrm{MHz}$. It was decided first to focus on the calibration methods and to use probes as transfer standards, each being measured at a number of locations in a round-robin intercomparison.

The pilot laboratory selected three electric, isotropic probes, two (to be referred to as 1 and 2) of a more common type with integrated readout, but with a fiber optic link, and a more sophisticated one (to be referred to as 3 ) with computer control and digital readout of the individual elements as well as their vector sum. Also, a magnetic probe (to be referred to as 4) was included.

In order to separate the field-sensing equipment from the electronic read-out, almost all instruments use fiber-optic links. The only exception was the magnetic field probe, for which VSL built such an extension.

Although tests were carried out to assure suitability for transport, some problems were encountered during the project. 


\section{Calibration Methods and Facilities}

\section{TEM Cell}

Most calibration laboratories prefer the use of the Crawfordtype TEM cell [2]. The closed structure allows a good characterization of the field. For calibration purposes, in general, the dimensions (septum width, outer conductor height, and width) are chosen to obtain a characteristic impedance of $50 \Omega$ in a symmetric TEM cell.

The field is calculated as follows:

$$
E=\left[\left(P_{\text {meas }} A Z_{0}\right) / d\right]^{\frac{1}{2}} \text { or } E=V / d
$$

where $P_{\text {meas }}$ is the RF output power measured after a power attenuator (attenuation $A$ ), $d$ is the septum height of the cell, $Z_{0}$ is the characteristic impedance, and $V$ is the measured voltage at the input or output of the cell.

The following laboratories have used this method.

- PTB (up to $125 \mathrm{MHz}$ ): The TEM cell has a septum height of $0.3 \mathrm{~m}$. The cell dimensions limit the upper frequency to about $160 \mathrm{MHz}$. The field strength is derived from power measurements and the cell geometry.

- NPL (up to $250 \mathrm{MHz}$, depending on probe): Its TEM cells (three commercial ones with different septum height ranging from 0.15 up to $0.92 \mathrm{~m}$ ) have a nominal characteristic impedance of $50 \Omega$. The cell losses are measured with the probe under test placed inside the cell at the measurement orientation using a network analyzer. The reflection coefficients $\left(S_{11}\right.$ and $S_{22}$ ) are measured with the cells empty. The frequency of the first cavity resonance is noted, and subsequent field strength measurements are carried out only below this frequency.

- STUK (up to $250 \mathrm{MHz}$ ): The TEM-300 cell (own design and construction) has a septum height of $0.25 \mathrm{~m}$ (upper part used). At higher frequencies (above $150 \mathrm{MHz}$ ), the internal fields have been mapped and the correction factors due to standing waves have been determined.

- Telecom Engineering (used by STUK for probe 4): This cell (own design and construction) has a septum height of $0.60 \mathrm{~m}$ (lower part used).

- VSL (up to $500 \mathrm{MHz}$ ): A set of two TEM cells, both with a cube midsection. In the larger cell (up to $150 \mathrm{MHz}$ : septum height $0.75 \mathrm{~m}$ ), a complete probe is usually placed inside the cell; in the smaller one (above $150 \mathrm{MHz}$ : septum height 0.15 $\mathrm{m}$ ), usually only the sensor element is exposed to radiation.

\section{Tapered TEM Cell and GTEM Cell}

In principle, a tapered TEM cell is just an extended taper of a normal TEM cell, only much longer and terminated with microwave absorbers to avoid reflection of the transmitted signal. The center conductor is suspended in the taper; it is electrically connected to the ground plane over the whole width of the septum end. At low frequencies, this might lead to significant reflections. In a GTEM cell (see, e.g., [3]), a distributed network of high-power resistive impedances connects the center conductor to the back wall of the taper, thus providing an impedance match as well as a high-power absorbing load. The field is calculated according to (1), using input power (or voltage). Correction factors for the inhomogeneity may be necessary.

As an alternative, a small calibrated probe can be used to measure the field strength at the reference point and relating this to the input power.

The following laboratories have used this method.

- PTB (up to $1000 \mathrm{MHz}$ ): The GTEM cell (1.5 m septum height) offers enough volume to expose complete field strength meters. The electric field strength is calculated from incident power. A correction factor is applied, based upon a scan of the field distribution with a calibrated $E$-field sensor.

- EMC Baden (up to $1000 \mathrm{MHz}$ ): In its GTEM cell, the field strength has been determined using the input voltage of the cell and the septum height of $1.20 \mathrm{~m}$ at the reference point.

- NPL (up to $1000 \mathrm{MHz}$ ): The NPL Tapered cell has a length of $3.4 \mathrm{~m}$, and has a pyramidal form with base dimensions of $1.8 \mathrm{~m} \times 1.2 \mathrm{~m}$ which is covered by 1.6 $\mathrm{m}$ pyramidal microwave absorber. One side of the outer conductor of the cell, normal to the septum, is removed to allow easy access for probes. In this way, the unwanted waveguide modes are radiated into the room and damped using blocks of microwave absorber. The effect on the TEM mode is corrected from the measurements with and without the side conductor mounted.

- SP (up to $125 \mathrm{MHz}$ ): In the SP-constructed cell, the input power/voltage is used to calculate the field strength. The field profile as a function of distance to the septum was determined to obtain a correction factor.

\section{Free-Space EM Field}

At higher frequencies, the method of creating a freespace electromagnetic field is preferred, usually in an (semi-)anechoic room. If suitable antennas are used, the electromagnetic field at a given position, with respect to the transmitting antenna, can be calculated using the input power $P$, the gain $G$ (or antenna factor) of the transmitting antenna, and the distance $d$, according to

$$
E=\left[\left(P G / 4 \pi Z_{0}\right) / d\right]^{\frac{1}{2}}
$$

where $Z_{0}$ is the wave impedance of free space $(120 \pi \Omega)$.

The gain may be calculated or may be obtained from a calibration laboratory. Far-field conditions are assumed; otherwise, correction factors have to be applied.

As an alternative, a small calibrated probe can be used to determine the field strength at the reference point directly.

The following laboratories have used this method.

- RFT used a shielded room (up to $1000 \mathrm{MHz}$ ), but not designed for calibration purposes. The probes have been located on a suitable table and irradiated by normal EMC antennas. A reference probe was placed next to the probe under test at a fixed distance. The read-out of the reference probe was used as field strength value.

- SP (250 up to $1000 \mathrm{MHz}$ ): Inside a semi-anechoic room, the field from a log-periodic antenna was controlled by measurement with precision dipoles (theoretical antenna factors). The antennas were connected to a spectrum analyzer with a preselector. 


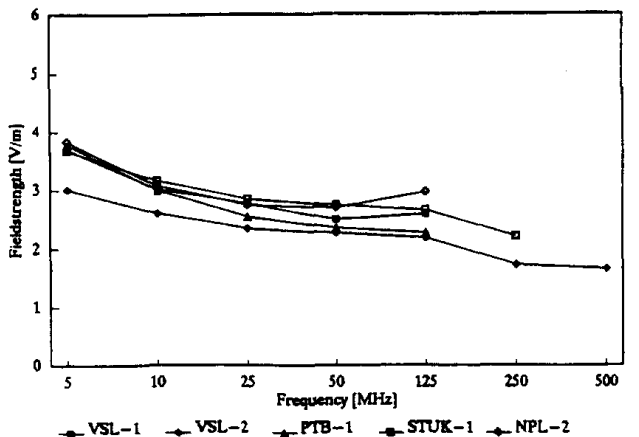

(a)

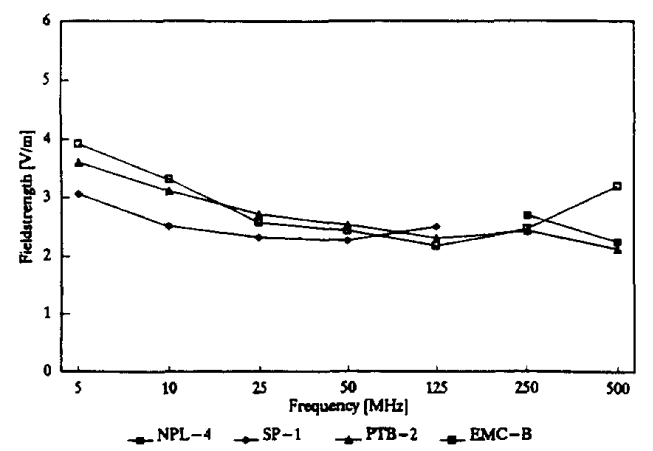

(b)

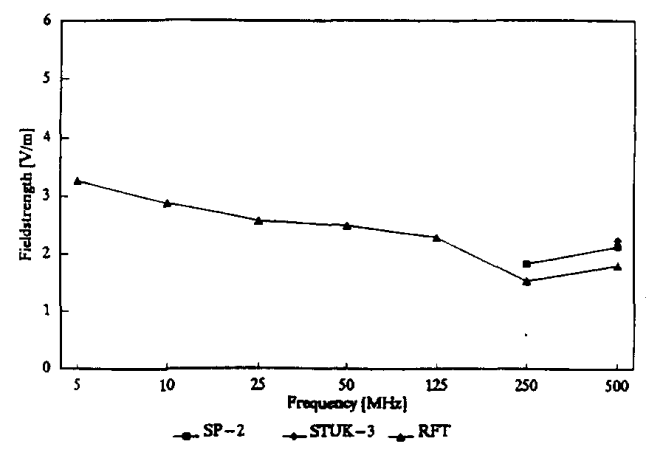

(c)

Fig. 1. Field strength necessary to obtain a $3 \mathrm{~V} / \mathrm{m}$ reading for probe 1 (the codes mentioned at the symbols refer to the specific measurement setup) for (a) TEM method, (b) T/G-TEM method, and (c) Open Area method.

- STUK(Facility at VTT: 500 up to $1000 \mathrm{MHz}$ ): A logperiodic antenna was used in an anechoic room with the probe under test at about $3 \mathrm{~m}$. Field strength is determined, using (2), from the input power to the antenna and calibrated gain values (manufacturer).

\section{Magnetic Field Generation}

All laboratories have used a TEM cell for magnetic sensor calibration, and not a commonly used method for calibrating a loop antenna based on the comparability of two equal loop antennas (induction method IEEE STD-302).

\section{RESULTS}

The pilot laboratory provided a set of guidelines to streamline the measurement process and data presentation. Each

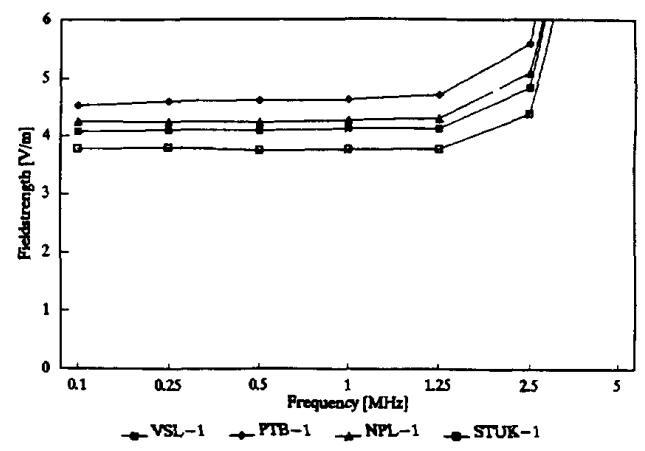

Fig. 2. Field strength necessary to obtain a $3 \mathrm{~V} / \mathrm{m}$ reading for probe 2 (the codes mentioned at the symbols refer to the specific measurement setup) using a TEM cell.

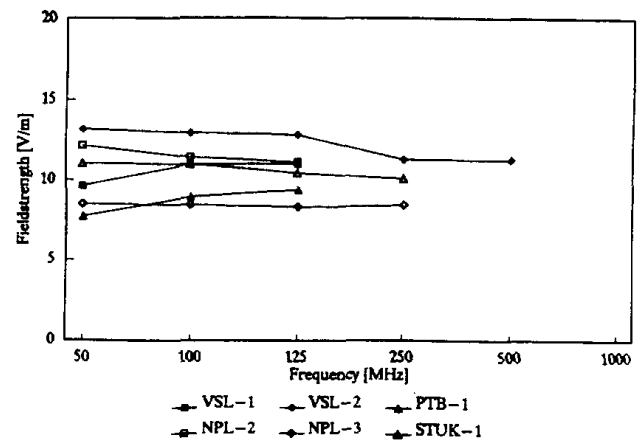

Fig. 3. Field strength necessary to obtain a $10 \mathrm{~V} / \mathrm{m}$ reading for probe 3 (the codes mentioned at the symbols refer to the specific measurement setup) using a TEM cell.

laboratory was asked to give the results in terms of field strengths generated for a certain indication of the instrument under calibration.

In principle, participants were asked to carry out their usual calibration procedure, preferably including a prescribed set of orientations. A detailed set of three pairs of orthogonal orientations was chosen to avoid ambiguity in the setup, based upon the directions of the $E$ field and the Poynting vector.

In Fig. 1, results for probe 1 are given for each calibration method: the measurement data, averaged over the prescribed set of orientations. The statistical spread appeared to be negligible compared to other contributions. In Figs. 2-4, similar results are presented for the other three probes, calibrated in TEM cells. Note that, due to practical reasons not all laboratories measured in all orientations.

In general, the smallest variation seems to be in the results obtained from calibrations performed in a TEM cell. The largest variation seems to be in the case of the Open Area method, but there is not sufficient data for comprehensive evaluation.

A more detailed investigation of the results from the three national standards laboratories, which carry out calibrations on a regular basis, leads to the following observations (see Table I for claimed uncertainties).

For orientations perpendicular to the electric field, the largest difference between the participants, found in the mea- 


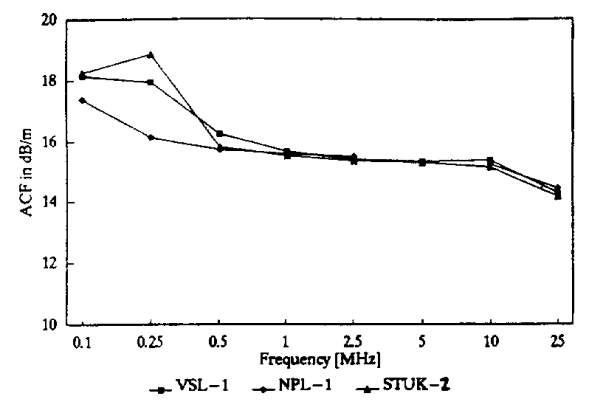

Fig. 4. Calculated antenna correction factor from the measured field strength necessary to obtain a $3 \mathrm{~V} / \mathrm{m}$ reading for probe 4 (the codes mentioned at the svmbols refer to the specific measurement setup) using a TEM cell.

TABLE I

Compil ation of the Systematic Uncertainties Quoted by Each Participant for Each Calibration Method (Ali. Given in dB and as $2 \sigma$-Values)

\begin{tabular}{|c|c|c|c|c|c|c|c|}
\hline $\begin{array}{l}\text { Laboratory } \\
\text { Method: }\end{array}$ & $\overline{V S L}$ & PTB & EMC-Baden & NPL & RFT & $\mathrm{SP}$ & STUK \\
\hline TEM & 0.4 & 0.3 & & 0.5 & & & 1.1 \\
\hline - t/GTEM & & 0.7 & 3.3 & 0.7 & & 3.2 & \\
\hline Open Area & & & & & 1.6 & 0.9 & 2.3 \\
\hline
\end{tabular}

surement data for probes 1 and 2 , is 0.07 and $0.02 \mathrm{~dB}$, respectively. However, for the orientations parallel to the electric field, significantly larger differences were obtained: 0.25 and $0.12 \mathrm{~dB}$, respectively.

For probe 3 , the agreement is worse, even in perpendicular orientations: up to $0.15 \mathrm{~dB}$. In this case, cable pick-up might explain the different results. Probe 4 (the magnetic field probe) is relatively large for most of the TEM and GTEM cells. Hence, the difference (1-2 dB) might be due to unaccounted sources of errors related to the size of the probe in such a restricted conducting volume. Also, positioning of the preamplifier and the performance of the fiberlink might be relevant.

Probes 1-3 should be isotropic, but on the basis of this exercise, they are not. For probes 1 and 3, there is a significant difference between orientations parallel and perpendicular to the $E$ field: $0.1-0.2$ and $2-3 \mathrm{~dB}$, respectively: the reading was higher when the axis of the probe was parallel to the electric field. Although there is too little correlation between the results, it seems as if a higher reading is obtained in a smaller cell.

During evaluation, VSL has investigated the probes in more detail. In a TEM cell, the probes have been placed under an angle of about $55^{\circ}$ to the electric field vector. Rotating the probes around the axis (a relative measurement) shows most clearly the individual responses of the receiving elements: these responses were almost identical, indicating a good isotropic behavior. As the coupling to the electric field should be minimal at perpendicular orientations, these results should be trusted.

\section{CONCLUSIONS}

Good absolute calibration (uncertainty on the order of 0.5 $\mathrm{dB}$ ) is realistic and should always contain an isotropy check. However, measurements on field strength meters (probes), positioned with the leads parallel to the electric field vector, lead to significant deviations between participants, and in comparison to other orientations. These differences are most likely due to interaction between probe and its immediate surroundings. Therefore, this orientation should be avoided.

Calibration of single magnetic loops using TEM/GTEM cells is a promising general method, and should be investigated in more detail.

It is quite clear that each type of probe exhibits its own problems. Therefore, regular exchange of information between users (EMC, radiation safety), calibration institutes, standardization bodies, and manufacturers should be promoted.

\section{REFERENCES}

[1] G. M. Teunisse and J. P. M. de Vreede, Calibration of Field Strength Meters. Luxembourg: Printing Office of the European Commission, 1994 (EUR 15890).

[2] M. L. Crawford, "Generation of standard EM-fields using TEM transmission cells," IEEE Trans. Electromagn. Compat., vol. EMC-16, no. 4, pp. 189-195, 1974.

[3] R. de Leo, T. Rozzi, C. Svara, and L. Zappelli, "Rigorous analysis of the GTEM cell," IEEE Trans. Microwave Theory Tech., vol. 39, pp. 488-500, Mar. 1991. 